\title{
Sex differences in outcomes following less-invasive left ventricular assist device implantation
}

\author{
Silvia Mariani ${ }^{1,2}$, Tong Li ${ }^{1}$, Karl Bounader ${ }^{1}$, Dietmar Boethig ${ }^{1}$, Alexandra Schöde ${ }^{1}$, Jasmin S. Hanke ${ }^{1}$, \\ Jana Michaelis ${ }^{1}$, L. Christian Napp ${ }^{3}$, Dominik Berliner ${ }^{3}$, Guenes Dogan ${ }^{1}$, Roberto Lorusso ${ }^{2}$, \\ Axel Haverich ${ }^{1}$, Jan D. Schmitto ${ }^{1}$
}

${ }^{1}$ Department of Cardiothoracic, Transplantation and Vascular Surgery, Hannover Medical School, Hannover, Germany; ${ }^{2}$ Cardio-Thoracic Surgery Department, Heart and Vascular Centre, Maastricht University Medical Centre (MUMC), Cardiovascular Research Institute Maastricht (CARIM), Maastricht, The Netherlands; ${ }^{3}$ Department of Cardiology and Angiology, Hannover Medical School, Hannover, Germany

Correspondence to: Prof. Dr. Jan D. Schmitto, MD, PhD, MBA. Dept. of Cardiothoracic, Transplant and Vascular Surgery, Medizinische Hochschule Hannover, Carl-Neuberg-Str. 1, 30625 Hannover, Germany. Email: Schmitto.Jan@mh-hannover.de.

\begin{abstract}
Background: Worse outcomes in women compared to men undergoing left ventricular assist device (LVAD) implantation remain an underestimated problem in heart failure (HF) patients. With device miniaturization, less-invasive LVAD implantation techniques have gained relevance, but their impact on outcomes in women is unknown. This study investigates sex-related differences in patients undergoing LVAD implantation through less-invasive procedures.

Methods: This retrospective single-center cohort study included patients who underwent isolated LVAD implantation between 2011 and 2018 through less-invasive techniques. Propensity score matching (PSM) was utilized to balance preoperative heterogeneity. Primary endpoint was two-year survival, and secondary endpoints included long-term survival, surgical outcomes and postoperative adverse events.

Results: Baseline analysis of 191 patients (females 18.3\%) showed differences in terms of age [female (median, 52; IQR, 47-61); male (median, 58.5; IQR, 49-66); $\mathrm{P}=0.005$ ], underlying diagnosis $(\mathrm{P}<0.001)$, INTERMACS profile $(\mathrm{P}=0.009)$, history of previous cardiac surgery $(\mathrm{P}=0.049)$ and preoperative creatinine values [female (median, 110; IQR, 71-146); male (median, 126; IQR, 9-168); P=0.049]. Over a follow-up of 460.68 patient-years, Kaplan-Meyer analysis showed better survival in females $(\mathrm{P}=0.027)$ and a similar probability of cardiac transplantation $(\mathrm{P}=0.288)$. After PSM, females showed higher needs for intraoperative fresh frozen plasma $(\mathrm{P}=0.044)$ and platelets $(\mathrm{P}=0.001)$ but comparable postoperative outcomes. No sexrelated differences were noticed regarding two-year outcomes, long-term survival and adverse events. LVADrelated infections remained the most common complication with males experiencing more pump infections than women $(\mathrm{P}=0.050)$.

Conclusions: Patients receiving less-invasive LVAD implantation do not show significant sex-related differences in short and long-term outcomes and survival. Prospective studies are needed to evaluate the role of less-invasive techniques in reducing sex-based disparities after LVAD implantation.
\end{abstract}

Keywords: Left ventricular assist device (LVAD); lateral thoracotomy; minimally invasive surgery; sex differences

Submitted Oct 05, 2020. Accepted for publication Feb 08, 2021.

doi: 10.21037/acs-2020-cfmcs-21

View this article at: http://dx.doi.org/10.21037/acs-2020-cfmcs-21

\section{Introduction}

The role of left ventricular assist devices (LVAD) as circulatory support in end-stage heart failure (HF) patients has been increasingly emphasized in the past decades (1).
Advancements in LVAD technical aspects, improvements in perioperative management and new surgical strategies have significantly contributed to the growing success of LVADs (2-5). Nevertheless, disparities can be noticed between 


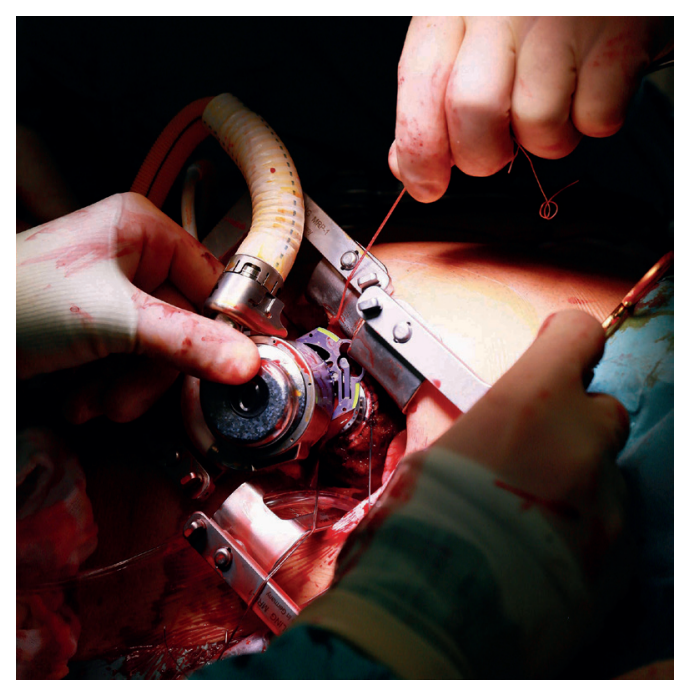

Figure 1 Intraoperative view of the left thoracotomy approach for LVAD implantation. The LVAD pump is being inserted into the left ventricular apex through left thoracotomy. LVAD, left ventricular assist device.

males and females (4-7). Indeed, women account for about $20 \%$ to $25 \%$ of the entire LVAD population (4-9) with a trend toward increasing LVADs in females (8). Significant heterogeneity exists in terms of underlying diagnoses $(10,11)$, severity of HF (12), and implanted devices (12). Moreover, contradictory reports exist regarding higher risks of stroke $(9,12-17)$, right $\mathrm{HF}$ (RHF) requiring right ventricular assist device (RVAD) implantation $(12,15,18,19)$, re-hospitalizations (9), bleeding and pump thrombosis (9) in women. Notwithstanding, the precise mechanisms underlying worse outcomes in women following LVAD implantation is still unclear, and no defined strategies have been described to address such a problem. In particular, the impact of different surgical techniques on LVAD outcomes in women is unknown. With the device miniaturization, a lateral thoracotomy for LVAD implantation (20) has gained relevance (2). Avoiding sternotomy and minimizing surgical trauma leads to reduced postoperative bleeding $(21,22)$, less transfusions (23), lower incidence of RHF (21,24-26), shorter hospital stay $(21-25,27-30)$ and lower costs $(31,32)$.

The present study investigates sex differences in a propensity score-matched population of patients undergoing LVAD implantation through less-invasive techniques.

\section{Methods}

\section{Study design and population}

This study retrospectively analyzed patients receiving LVAD implantation at a single high-volume center from January 2011 to January 2018. All candidates were adults undergoing isolated LVAD implantation through less-invasive techniques. Only primary implantations were included while biventricular assist devices or combined surgeries were excluded. All patients were categorized based on their biologically determined sex (male or female) (33-35). Patients attended follow-up visits every three months for the time of LVAD support and completed the followup for survival in August 2020. Demographic and clinical variables were collected. Primary endpoint was two-year survival. Secondary endpoints were overall survival, adverse events defined according to INTERMACS classifications, intensive care unit (ICU) and hospital stay, intraoperative transfusions, postoperative mechanical circulatory support (MCS), ventilation time, pump exchange rates, and explant for recovery and transplantation.

Data collection and analysis were approved by the institutional review board (Study ID number: 8642_BO_ K_2019). Every patient who prospectively entered the LVAD program at the study institution and was included in this study signed a consent form for data use for research purposes.

\section{Surgical approaches and clinical management}

In the study time, all included patients who received a HeartWare HVAD System (Medtronic, Inc., Minneapolis, USA) or HeartMate3 (Abbott, Chicago, USA) underwent LVAD implantation through the "Hannover technique" (Figure 1) (20). Briefly, a J-shaped hemisternotomy up to the second or third intercostal space and a left anterolateral thoracotomy were performed above the left ventricular (LV) apex. The correct spot for the LV core was identified by gentle digital compression under echocardiographic monitoring. The apical ring was sewn to the heart, cardiopulmonary bypass $(\mathrm{CPB})$ was started, and the LV apex was cored, allowing inspection of the LV cavity. The LVAD pump was then inserted. The driveline and outflow graft were tunneled and the outflow graft anastomosis completed. Gradual weaning from CPB and pump speed adjustment were performed. All patients who received a HeartMate II 
(Abbott, Chicago, USA) underwent surgery through the subcostal incision as previously described (36).

Preoperative evaluation and patient selection for both males and females followed the principles previously described for LVAD implantation through lateral thoracotomy (37). Postoperative management was at the discretion of the attending physicians, and no different protocols were applied in males and females. Generally, the international guidelines for postoperative management of patients receiving LVAD implantation $(38,39)$ and the specific indications for patients undergoing surgery through lateral thoracotomy were followed (37). Intravenous heparin was started once chest tube drainage was less than $50 \mathrm{~mL} /$ hour for three hours. The heparin dose was increased over two days to reach a partial thromboplastin time of 55 to 65 seconds. Aspirin and vitamin $\mathrm{K}$ antagonists were started once the patient was able to take oral medications and continued throughout support, with a target international normalized ratio of 2.0 to 3.0. Blood pressure was measured by Doppler sonography and mean target blood pressure was 60 to $65 \mathrm{mmHg}$.

\section{Statistical analysis}

Demographic and clinical variables are expressed as count (with percentage) for categorical variables and mean ( \pm standard deviation) or median (minimum-maximum) for continuous variables after evaluation for normality. Group comparisons were made with McNemar's test and the Wilcoxon rank-sum test for the continuous variables and Pearson's $\chi^{2}$ or Fisher's exact test for categorical variables. Kaplan-Meier survival analysis and Log-Rank test were performed to determine differences in survival. Events were censored at the end of follow-up or in case of transplantation and explant. Cumulative incidence of transplantation was analyzed. A two-tailed $\mathrm{P}$ value of $\leq 0.05$ was considered significant. Analyses were performed using SPSS 24.0 (IBM, Armonk, New York, USA), Prism 8.0 (GraphPad Software, Inc., San Diego, USA) and R 3.6.3 (R Foundation for Statistical Computing, Vienna, Austria).

\section{Propensity score matching}

Data analysis was performed to compare male and female patients. Based on significant baseline differences and the evaluation of factors that could have influenced outcomes, propensity score (PS) matching was performed. The PS was calculated via non-parsimonious multivariable logistic regression model based on age, body mass index, implanted LVAD device type, HF etiology, INTERMACS profile, diabetes, myocardial infarction, cardiothoracic surgery history, preoperative MCS, LV ejection fraction and serum creatinine. Patients were matched 1:1 using a greedy matching algorithm (nearest match without replacement) based on each patient's PS with a caliper width of $10 \%$ of the standard deviation of the PS's logit.

\section{Results}

\section{Overall population: baseline characteristics}

A total of 191 patients were enrolled in this study, with thirty-five women accounting for $18.32 \%$ of the entire cohort. The baseline analysis showed significant differences in age, underlying diagnosis, INTERMACS profile, history of previous cardiac surgery and preoperative creatinine values (Table 1). Women were younger and more frequently classified as INTERMACS profile 1 . The cardiac diagnosis differed between groups with women being affected by a higher prevalence of dilated cardiomyopathy and a few post-partum cardiomyopathy cases $(n=5)$. Men had more frequently a history of previous cardiac operations and higher values of serum creatinine.

\section{Overall population: survival analysis}

In the pre-matched cohort, 460.68 patient-years $(168,263$ days) were analyzed. The median duration of support was 1,080 days $(1-3,190$ days $)$, with similar values $(\mathrm{P}=0.082)$ between females $(962 ; 1-3,093)$ and males $(1,281 ; 2-3,190)$. Kaplan-Meyer analysis (Figure 2) showed better survival in favor of women $(\mathrm{P}=0.027)$ and the similar probability of cardiac transplantation $(\mathrm{P}=0.290)$. When analyzing patients' status at two years, higher rates of alive and transplanted women were observed (Figure 3). A subgroup analysis of INTERMACS 1 patients $(\mathrm{n}=34)$ showed no differences in the probability of survival $(\mathrm{P}=0.099)$ (Figure $\mathrm{S} 1)$.

\section{Propensity score matched population: baseline characteristics and perioperative outcomes}

After matching, fifty-two patients with comparable preoperative characteristics were selected. The overall average age at implantation was $53.3 \pm 12.0$ years, and the median body mass index was $24.3 \mathrm{~kg} / \mathrm{m}^{2}\left(18.6-43.4 \mathrm{~kg} / \mathrm{m}^{2}\right)$. Dilated cardiomyopathy was the most common diagnosis $(n=32,61.5 \%)$, two women were diagnosed with peripartum 


\begin{tabular}{|c|c|c|c|}
\hline Variables & Females $(n=35)$ & Males $(n=156)$ & $P$ value \\
\hline Age, years & $52[25-75]$ & $58.5[18-79]$ & 0.005 \\
\hline $\mathrm{BMI}, \mathrm{kg} / \mathrm{m}^{2}$ & $24.12[18.56-43.42]$ & $25.95[16.48-42.92]$ & 0.283 \\
\hline Device, n (\%) & & & 0.391 \\
\hline HVAD & $34(97.1)$ & $144(92.3)$ & \\
\hline HM3 & $0(0)$ & $8(5.1)$ & \\
\hline HM II & $1(2.9)$ & $4(2.6)$ & \\
\hline Etiology, n (\%) & & & $<0.001$ \\
\hline $\mathrm{DCM}$ & $19(54.3)$ & $77(49.4)$ & \\
\hline ICM & $9(25.7)$ & $75(48.1)$ & \\
\hline Others & $7(20.0)$ & $4(2.6)$ & \\
\hline INTERMACS, n (\%) & & & 0.009 \\
\hline Profile 1 & $9(25.7)$ & $25(16.0)$ & \\
\hline Profile 2 & $0(0)$ & $21(13.5)$ & \\
\hline Profile 3 & $9(25.7)$ & $48(30.8)$ & \\
\hline Profile 4 & $15(42.9)$ & $62(39.7)$ & \\
\hline Profile 5-7 & $2(5.8)$ & $0(0)$ & \\
\hline Diabetes, n (\%) & $4(11.4)$ & $33(21.2)$ & 0.240 \\
\hline Previous myocardial infarction, $\mathrm{n}(\%)$ & $6(17.1)$ & $14(9.0)$ & 0.216 \\
\hline Previous cardiac surgery, n (\%) & $7(20.0)$ & $60(38.5)$ & 0.049 \\
\hline Pre-operative MCS, n (\%) & $9(25.7)$ & $24(15.4)$ & 0.148 \\
\hline Preoperative LVEF, \% & 20 [5-35] & 20 [5-38] & 0.154 \\
\hline Preoperative serum creatine, $\mathrm{mmol} / \mathrm{L}$ & $110[44-479]$ & $126[49-864]$ & 0.049 \\
\hline
\end{tabular}

Data are expressed as $\mathrm{n}(\%)$, mean \pm standard deviation or median [min-max] as appropriate. BMI, body mass index; DCM, dilated cardiomyopathy; HM II, HeartMate II; HM3, HeartMate3; HVAD, HeartWare; LVEF, left ventricular ejection fraction; MCS, mechanical circulation support.

cardiomyopathy and two men suffered from myocarditis. The majority of patients were NYHA profile $4(n=48$, $92.3 \%$ ) and INTERMACS profile 1 was observed in seven cases $(13.5 \%)$. The median ejection fraction was $20 \%$ (5-35\%), and men showed higher values of LV end-diastolic diameters. Preoperative MCS was required in six patients $(11.5 \%)$. Further baseline characteristics are presented in Table 2.

The majority of patients received implantation of an HVAD both in the un-matched and matched population. No female received a HeartMate3 (Abbott, North Chicago, IL, USA) device implantation and, thus, it was automatically excluded from PS matching analysis. All operations were accomplished through less-invasive approaches, and none required conversion to conventional sternotomy. Median operation time and CPB time were 209.5 minutes (120410 minutes) and 53.5 minutes (31-151 minutes), respectively. One male refused the use of $\mathrm{CPB}$ due to religious reasons. A significant difference was observed for intraoperative transfusions: females received a higher amount of fresh frozen plasma and platelets (Table 3) but a comparable amount of packed red cells. Despite this, no significant differences were noticed in postoperative rethoracotomies which accounted for $3.8 \%$ of cases. Similarly, 
Overall Population before Propensity Score Matching

A

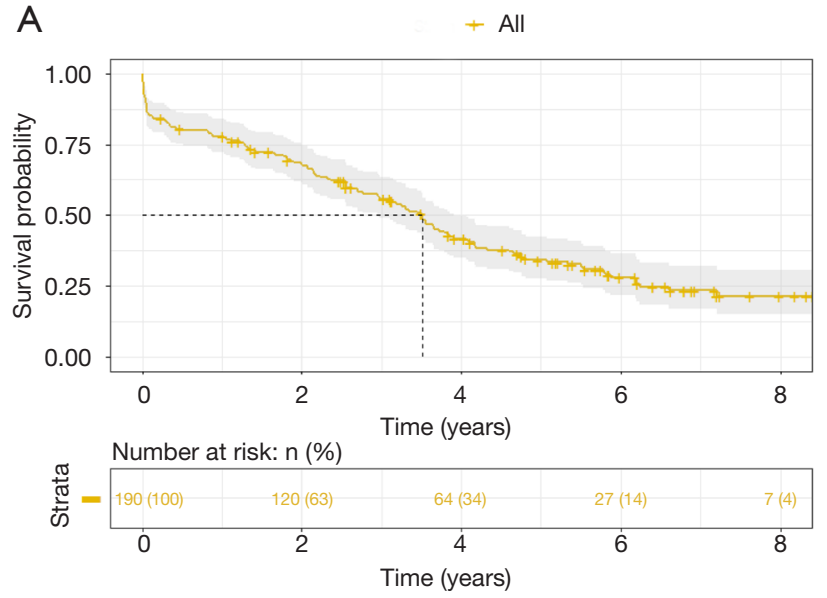

C

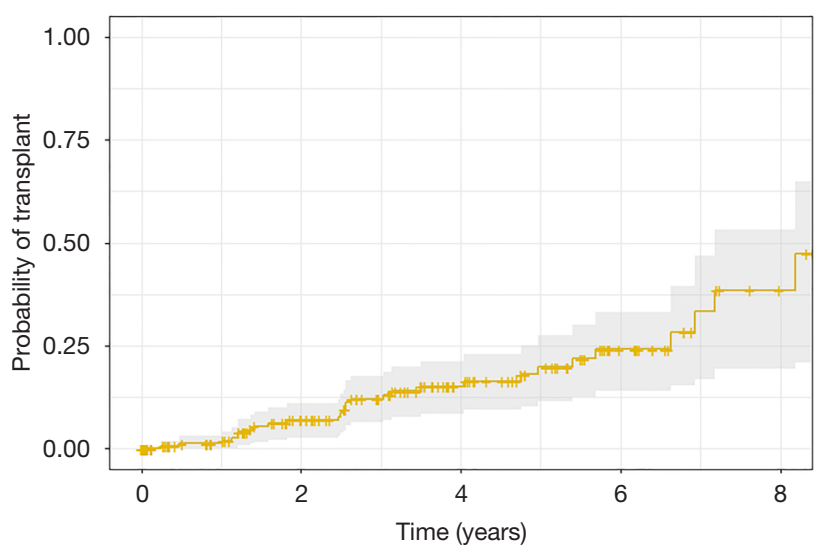

B
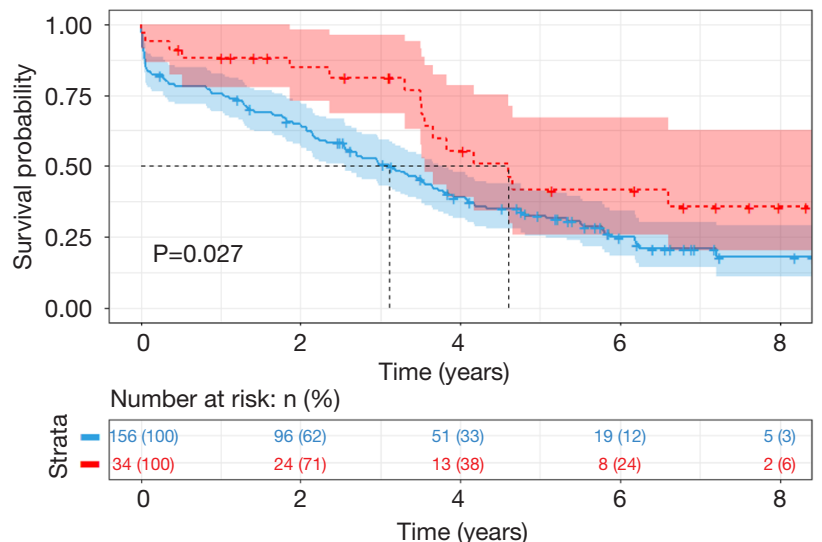

D

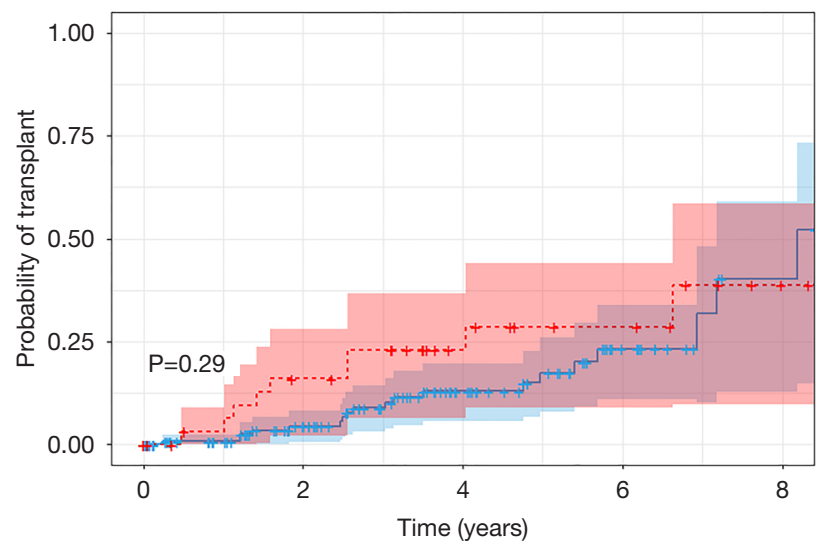

Figure 2 Kaplan-Meier survival analysis of the overall cohort (A) and by sex (B) before propensity score matching. Probability of transplant of the overall cohort (C) and by sex (D) is shown.

postoperative RHF and the need for extracorporeal membrane oxygenation (ECMO) were comparable. Seven patients required postoperative ECMO: $62.5 \%$ of them (three females and two males) were already on ECMO before surgery, and one patient received ECMO three days after surgery for acute pump thrombosis. One patient was treated with temporary RVAD (percutaneous cannulation of the right atrium and pulmonary artery) for ten days, and one patient required a durable RVAD. Mechanical ventilation times, respiratory failure rates, ICU stay and hospital stay were also comparable between groups.

\section{Propensity score matched population: survival analysis}

Patients were followed-up for 320.47 patient-years
(117,051 days); only one patient was lost to follow-up. The median duration of support was 1,280 days (14-3,202 days), with similar values $(\mathrm{P}=0.258)$ between females $(1,308$; 22-3,202) and males $(1,105 ; 14-2,987)$. Overall, fourteen patients $(26.9 \%)$ underwent cardiac transplantation during follow-up with comparable probabilities for women and men $(\mathrm{P}=0.630)$. Eight females $(30.8 \%)$ and four men $(15.4 \%)$ remained on the device until the end of the follow-up (Figure 4).

In-hospital survival was $94.2 \%$ (females: $96.2 \%$; males: $92.3 \% ; \mathrm{P}=0.500$ ). At two years, mortality was $7.7 \%$ in women and $24 \%$ in males, $15.4 \%$ of females and $12 \%$ of males received transplantation and $76.9 \%$ of women and $64 \%$ of men were alive on device (Figure 3; $\mathrm{P}=0.260)$. Kaplan-Meier analysis and cumulative incidence 

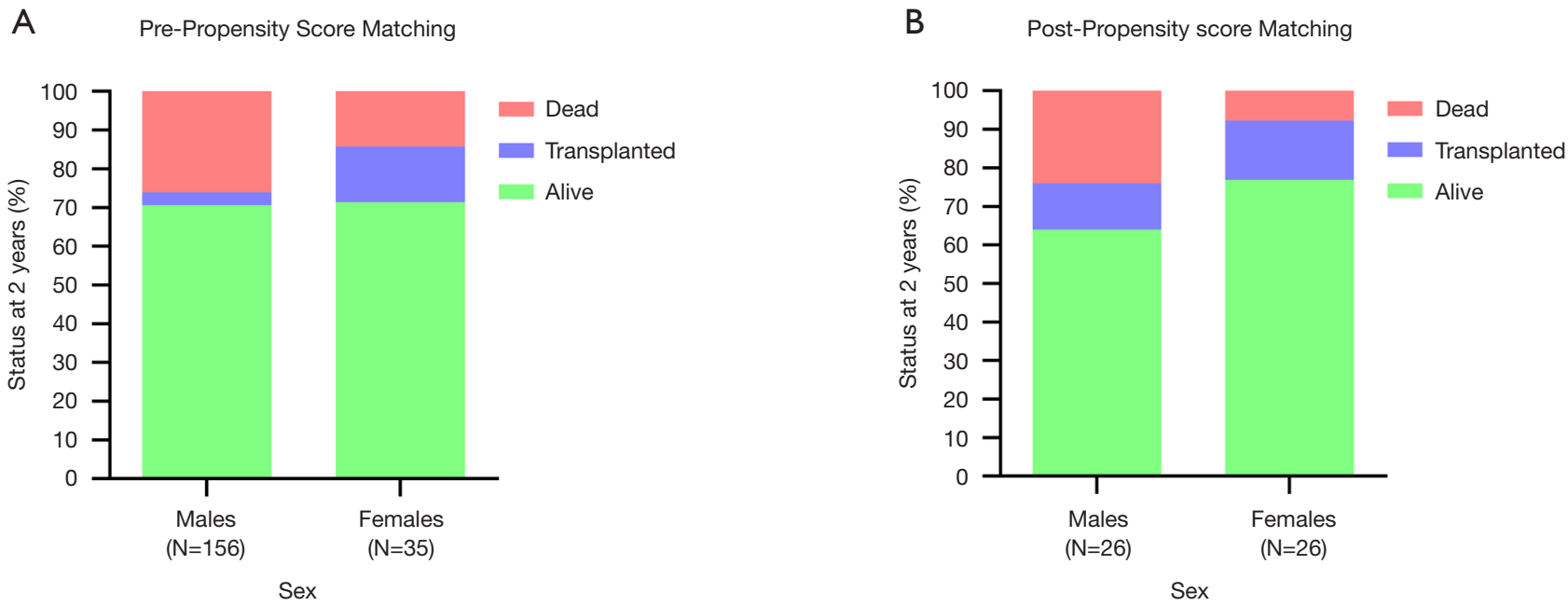

Figure 3 Outcomes at 2 years follow-up in patients' overall cohort before propensity score matching (A) and after propensity score matching (B).

of transplantation were comparable between groups (Figure 4).

\section{Propensity score matched population: adverse events}

Pump thrombosis and LVAD-related infections were the most common complications (Figure 5). In detail, pump thrombosis occurred in eight women (30.8\%, 0.1307 events/ patient-year, EPPY) and seven men (26.9\%, 0.0876 EPPY; $\mathrm{P}=0.760$ ) with two patients in each group experiencing repeated pump thrombosis (7.7\%). In all these cases except one woman, a pump exchange was required. Overall, seven women $(26.9 \%, 0.1188 \mathrm{EPPY})$ and ten men $(38.5 \%$, $0.1266 \mathrm{EPPY}$ ) underwent pump exchange through lessinvasive surgery and none of them required conventional sternotomy $(\mathrm{P}=0.375)$. While thrombosis was the reason for pump exchange in all women, three men underwent surgery for the pump pocket infection. Indeed, pump infection was diagnosed more often in men $(n=7,26.9 \%$, $0.0682 \mathrm{EPPY})$ than in women $(\mathrm{n}=1,3.8 \%, 0.0119 \mathrm{EPPY}$; $\mathrm{P}=0.050)$ while driveline infections were similar between groups [females ( $\mathrm{n}=11,42.3 \%, 0.1307 \mathrm{EPPY})$; males $(\mathrm{n}=12$, $46.2 \%, 0.1363 \mathrm{EPPY}) ; \mathrm{P}=0.780]$. The occurrence of strokes, gastrointestinal bleeding and the need for long-term dialysis were also comparable between groups.

\section{Discussion}

This is the first study investigating sex differences in HF patients' outcomes after LVAD implantation through less-invasive techniques. The analysis of the un-matched population, including $18.3 \%$ of women, showed significant baseline differences in age, underlying diagnosis, INTERMACS profile, history of previous cardiac surgery and preoperative kidney function. Despite females being more often in INTERMACS 1 status, their survival probability resulted better with higher rates of alive and transplanted women at two years. After PS matching, women showed higher needs for intraoperative transfusions but comparable postoperative outcomes. No sex-related differences were noticed in two-year results, long-term survival and adverse events. LVAD-related infections remained the most common complication with males experiencing more pump infections.

$\mathrm{HF}$ is a growing medical burden worldwide, with nearly $40-50 \%$ of cases diagnosed in women $(40,41)$. Nevertheless, several studies demonstrated that advanced HF therapies remain underused in females $(6,7)$, even though the number of women undergoing LVAD implantation has increased with the introduction of continuous-flow devices (8). Moreover, women supported by LVADs have higher mortality rates and lower heart transplantation chances $(7,14)$. Some studies suggested that women are more likely to be in cardiogenic shock at the time of LVAD implantation (12), they experience higher rates of perioperative RHF requiring RVADs $(12,15,18,19)$ and they have a higher risk for neurological and bleeding complications $(12-14,16,17)$. Despite the awareness of the 
Table 2 Baseline characteristics after propensity score matching

\begin{tabular}{llll}
\hline Variables & Females $(n=26)$ & Males $(n=26)$ & $P$ value \\
\hline Age, years & $52.59 \pm 12.90$ & $54.65 \pm 11.65$ & 0.423 \\
BMI, kg/m ${ }^{2}$ & $24.24[18.56-43.41]$ & $24.22[18.75-42.61]$ & 0.869 \\
Device, $\mathrm{n}(\%)$ & & & 0.755 \\
HVAD & $25(96.2)$ & $25(96.2)$ \\
HM II & $1(3.8)$ & $1(3.8)$
\end{tabular}

$\begin{array}{llc}\text { Etiology, } \mathrm{n}(\%) & \\ \text { DCM } & 17(65.4) & 15(57.7) \\ \text { ICM } & 7(26.9) & 9(34.6) \\ \text { Others } & 2(7.7) & 2(7.7)\end{array}$

$\begin{array}{lll}\text { NYHA, } n(\%) & & \\ \text { Class } 3 & 1(3.8) & 3(11.5) \\ \text { Class } 4 & 25(96.2) & 23(88.5)\end{array}$

\begin{tabular}{|c|c|c|c|}
\hline INTERMACS, n (\%) & & & 0.665 \\
\hline Profile 1-2 & $4(15.4)$ & $3(11.5)$ & \\
\hline Profile 3-4 & $22(84.6)$ & $23(88.5)$ & \\
\hline Profile 5-7 & $0(0)$ & $0(0)$ & \\
\hline History of stroke, n (\%) & $3(11.5)$ & $0(0)$ & 0.235 \\
\hline COPD, n (\%) & $1(3.8)$ & $2(7.7)$ & 1.000 \\
\hline Diabetes, n (\%) & $4(15.4)$ & $2(7.7)$ & 0.668 \\
\hline Previous myocardial infarction, n (\%) & $3(11.5)$ & $3(11.5)$ & 1.000 \\
\hline Previous cardiac surgery, $\mathrm{n}(\%)$ & $7(26.9)$ & $6(23.1)$ & 1.000 \\
\hline ICD implantation, $\mathrm{n}(\%)$ & $19(73.1)$ & $20(76.9)$ & 1.000 \\
\hline Pre-operative MCS & $4(15.4)$ & $2(7.7)$ & 0.668 \\
\hline Preoperative serum creatine, $\mathrm{mmol} / \mathrm{L}$ & $106[59-479]$ & 118 [65-360] & 0.407 \\
\hline \multicolumn{4}{|l|}{ Preoperative echocardiography } \\
\hline LVEF, \% & $20[13-25]$ & $17[10-35]$ & 0.451 \\
\hline LVEDD, mm & $66[50-99]$ & $73[59-110]$ & 0.006 \\
\hline \multicolumn{4}{|l|}{ Mitral valve regurgitation, $\mathrm{n}(\%)^{*}$} \\
\hline 0 & 4 & 6 & \\
\hline$|-| \mid$ & 14 & 17 & \\
\hline$>11$ & 5 & 1 & \\
\hline \multicolumn{4}{|l|}{ Tricuspid valve regurgitation, $\mathrm{n}(\%)^{\star}$} \\
\hline 0 & 3 & 5 & \\
\hline I-II & 18 & 16 & \\
\hline$>11$ & 2 & 3 & \\
\hline
\end{tabular}

Data are expressed as $\mathrm{n}(\%)$, mean \pm standard deviation or median [min-max] as appropriate. * preoperative echocardiography reporting data on valve function were available for 23 females and 24 males. BMI, body mass index; DCM, dilated cardiomyopathy; HM II, HeartMate II; HVAD, HeartWare; LVEF, left ventricular ejection fraction; MCS, mechanical circulation support. 


\begin{tabular}{|c|c|c|c|}
\hline Variables & Females $(n=26)$ & Males $(n=26)$ & $P$ value \\
\hline Operation time, $\min$ & $229.92 \pm 46.71$ & $212.43 \pm 59.64$ & 0.609 \\
\hline Cardiopulmonary bypass time, min & $51[32-141]$ & $54[31-151]$ & 0.295 \\
\hline \multicolumn{4}{|l|}{ Intraoperative blood products, $U$} \\
\hline Packed red cells & $3[0-16]$ & $2[0-9]$ & 0.083 \\
\hline Fresh frozen plasma & $4[1-16]$ & $2[0-13]$ & 0.044 \\
\hline Platelets & $2[0-3]$ & $1[0-4]$ & 0.001 \\
\hline Re-thoracotomy for bleeding, $\mathrm{n}(\%)$ & $1(3.8)$ & $1(3.8)$ & 0.118 \\
\hline Post-operative ECMO, n (\%) & $4(15.4)$ & $3(11.5)$ & 0.500 \\
\hline Post-dialysis, n (\%) & $5(19.2)$ & $3(11.5)$ & 0.352 \\
\hline Post-operative RHF, n (\%) & $4(15.4)$ & $1(3.8)$ & 0.175 \\
\hline RVAD, n (\%) & $2(7.7)$ & $0(0)$ & 0.245 \\
\hline Mechanical ventilation time, hours & $22[11-888]$ & 24 [15-283] & 0.082 \\
\hline Respiratory failure, n (\%) & $4(15.4)$ & $3(11.5)$ & 0.500 \\
\hline ICU stays, days & $4[1-41]$ & $4[1-46]$ & 0.860 \\
\hline Hospital stays, days & 26 [6-83] & 25 [12-140] & 0.795 \\
\hline Hospital death, n (\%) & $1(3.8)$ & $2(7.7)$ & 0.500 \\
\hline
\end{tabular}

Data are expressed as $\mathrm{n}(\%)$, mean \pm standard deviation or median [min-max] as appropriate. ECMO, extracorporeal membrane oxygenation; ICU, intensive care unit; RHF, right heart failure; RVAD, right ventricular assist device.

problem, little is known about the underlying causes of these differences, and no established strategies to address this clinical question are available. In particular, the impact of surgical approaches on LVAD outcomes in women is still unknown. It has been demonstrated that less-invasive techniques can significantly modify LVAD outcomes (21-32). However, since women are often under-represented in clinical studies, little is known about sex differences in outcomes following less-invasive LVAD implantation.

This study included 191 patients receiving LVAD implantation through less-invasive techniques at a single center from 2011 to 2018. Baseline analysis showed that included women were younger, with less ischemic cardiomyopathy and more often in severe cardiogenic shock as previously described in the literature $(7,8)$. Despite these differences, we observed an overall better survival in women which is in contrast with previous studies (6,11-14). In particular, good survival was noticed in INTERMACS 1 women. Indeed, it has been demonstrated that the use of a lateral thoracotomy in INTERMACS 1 patients is associated with the protection of the RV, reduced rethoracotomies and lower mortality (22). Generally, the use of a lateral thoracotomy for LVADs allows the pericardium to be left closed, thereby leading to further stabilization of RV function, including avoidance of RV distortion and dilatation (20,24). Moreover, reduced surgical trauma can prevent postoperative bleeding which is known as risk factor for arrhythmias and RHF $(11,12,15)$. This allows patients to overcome the acute postoperative phase and enter the follow-up period.

To reduce the influence of baseline differences on postoperative outcomes, a PS matched analysis was performed. After matching, comparable results in survival and transplantations were observed. Contrarily, several previous studies described higher in-hospital mortality rates in women, ranging from $9 \%$ to $14.5 \%(6,12,42)$. Higher postoperative mortality has been directly correlated to a $5 \%$ to $42 \%$ incidence of RHF $(11,12,15,18)$ and a $3 \%$ to $50 \%$ incidence of RVAD implantation in females $(11,12,15,19)$. Indeed, in women with a need for LVAD and temporary 


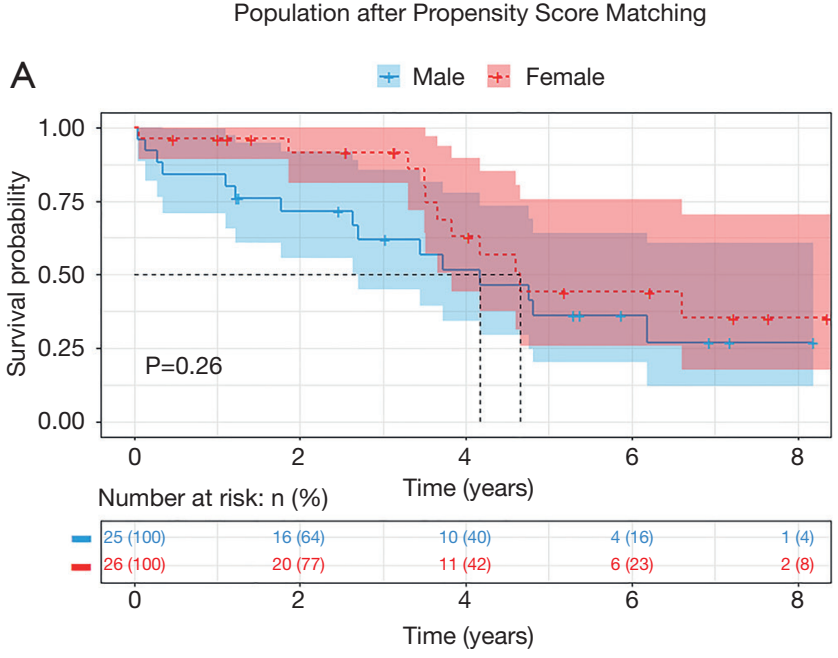

B

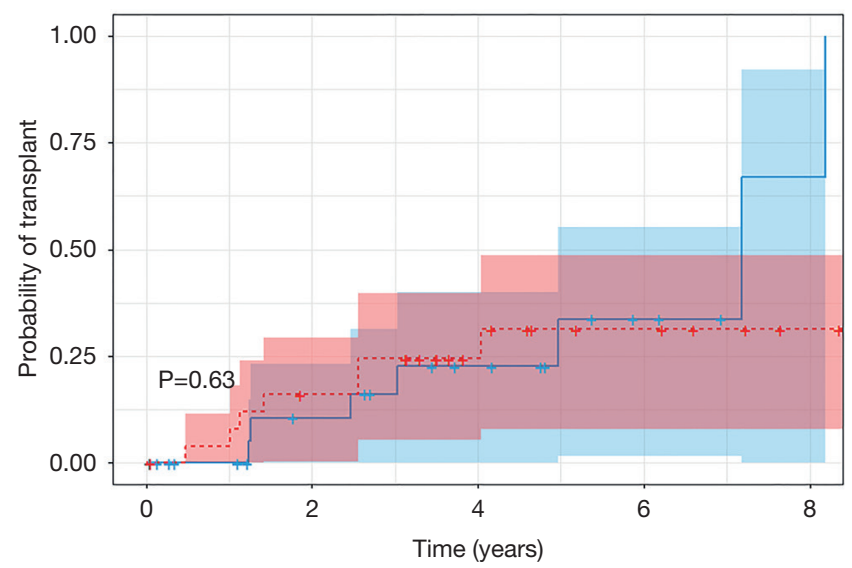

Figure 4 Kaplan-Meier survival curves and by sex (A) after propensity score matching. Probability of transplant by sex (B) is shown.

RVAD support, the probability of death is $38 \%$ at one year compared with $25 \%$ for women undergoing isolated LVAD support (12). In our matched population, $15.4 \%$ of women were diagnosed with RHF, and 7.7\% required RVAD.

A further factor for optimal short-term outcomes is the prevention of re-thoracotomies. In this study, despite $25 \%$ of patients having undergone previous cardiac surgery, only $3.8 \%$ of cases required a re-thoracotomy. Again, these data mirror the available literature on less-invasive LVAD implantation where rates of re-operations for bleeding are $0 \%$ to $13.6 \%(21,22,43)$. Minimization of bleeding allows early anticoagulation and prevention of pump thrombosis, while the low transfusion rate may decrease systemic inflammation and sensitization.
Notwithstanding, we noticed a higher intraoperative use of fresh frozen plasma and platelets in women, which might explain sex-related differences in hemostasis and thrombosis pathophysiological mechanisms $(44,45)$. This hypothesis surely deserves further investigations while literature lacks extensive studies investigating the different coagulative patterns in males and females undergoing cardiac surgery. Overall, there is an urgent need for studies addressing specific sex-based protocols for postoperative management of LVAD patients, including transfusion strategies, HF medications and social and psychological aspects (46).

Further major concerns regarding women's outcomes relate to higher rates of neurological events $(13,14)$. Several studies report a higher risk of strokes in women $(16,17)$ despite a lower frequency of traditional risk factors such as hypertension and atrial fibrillation (17). In this study, over a follow-up time of 320.47 patient-years, $15.4 \%$ (0.0475 EPPY) and 19.2\% (0.0832 EPPY) of women experienced an ischemic or hemorrhagic stroke, with similar incidences in men. Our results differ from previous studies because most of our patients received implantation of a centrifugal continuous-flow LVAD. In contrast, most studies reporting higher stroke rates analyzed patients supported with HeartMate II $(13,16,17)$. An examination of survival by sex in the ADVANCE BTT+CAP trial showed comparable stroke rates between men and women (11). Similarly, sex had no impact in the six-month endpoint outcomes (including disabling strokes) of the MOMENTUM3 trial (47). Therefore, these results should be interpreted considering the influence of different anticoagulation strategies, implanted devices and patients' compliance over time.

An interesting variability has been reported in terms of infections. Our study showed comparable rates of driveline infections but a higher incidence of severe pump infections in men, requiring pump exchange or leading to further complications and death. Interestingly, a direct correlation with the thoracotomy wound postoperative infection could not be established, and $57.1 \%$ of males experiencing pump infection received this diagnosis over three years after implantation. While literature reports similar infection rates in males and females, very few studies present followup times exceeding the two years. Thus, they might have underestimated the incidence of such a complication. Further investigations are needed to clarify the reasons for such a difference. Several hypotheses might be formulated, including the roles of social variables as well as compliance factors. 


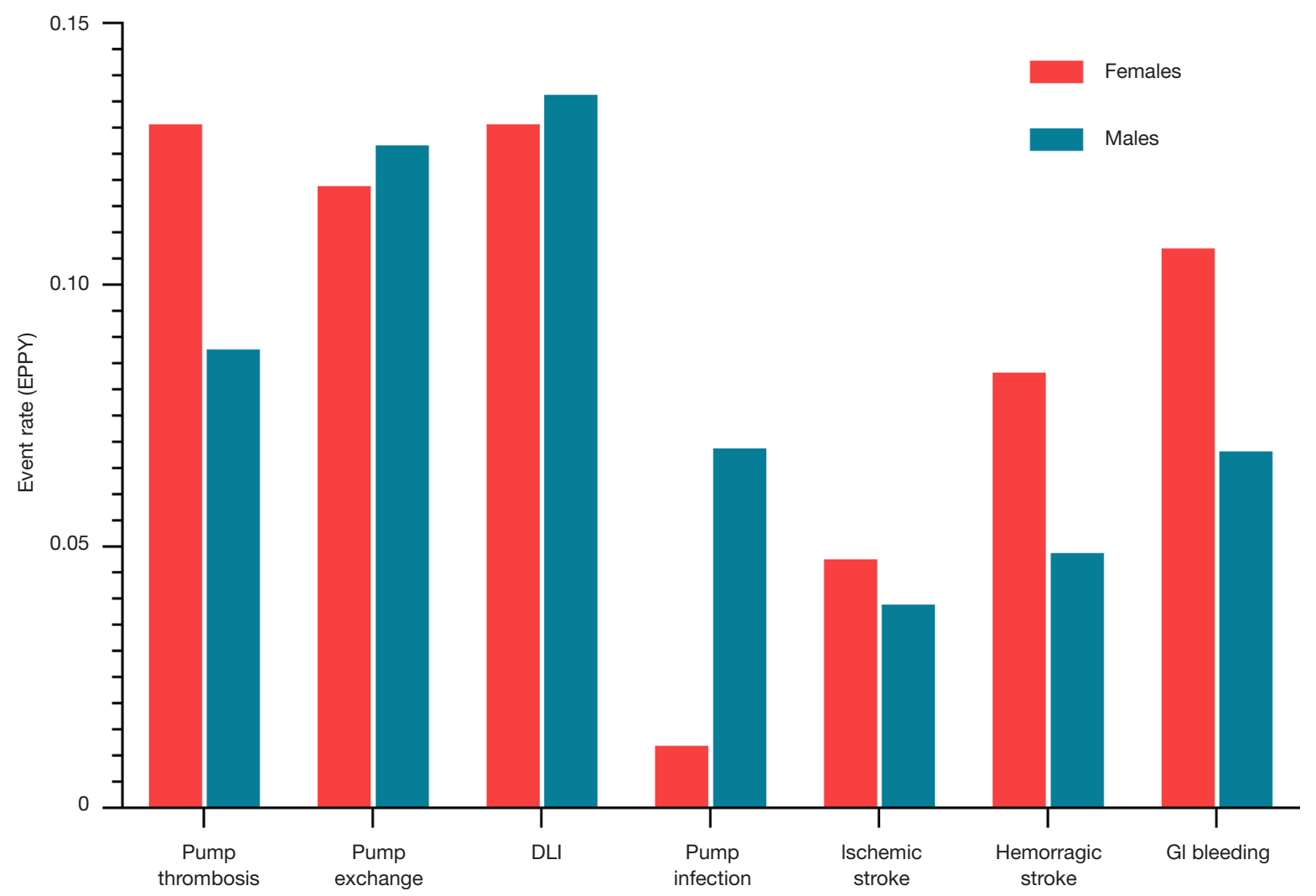

Figure 5 Adverse events in females and males after propensity score matching. DLI, driveline infections; EPPY, events/patient-year; GI, gastrointestinal.

\section{Limitations}

This study needs to be viewed in the context of several limitations. This was not a prospectively randomized study. All patients were operated by the same surgical team that also decided when to use less-invasive techniques. As with most single-center retrospective studies, the number of patients and study power is limited and further generalization of results should be considered carefully. Moreover, included patients received LVAD implantation between 2011 and 2018. Consequently, results are hardly comparable to the most recent studies which benefit from new therapeutic strategies and increased clinical experience. Similarly, few patients received a HeartMate 3 through lateral thoracotomy between June 2014 and January 2018 at our institution. HeartMate 3 is not considered as a contraindication for less invasive LVAD implantation in both males and females, although a slightly longer incision might be necessary due to the larger size of this pump (Figure 1) (37). However, between June 2014 and January
2018 a learning curve for HeartMate3 was required, and less-invasive surgeries started on a later stage compared to HeartMate3 implantations through conventional sternotomy. Adverse events were reported by treating physicians and were not monitored for completeness or adjudicated by an objective committee. While this did not affect survival, we cannot exclude the under-reporting of other adverse events, especially for gastrointestinal bleeding. All implantations were performed at a single high-volume LVAD center specialized in the use of lessinvasive techniques. Thus, results might have been affected by the institutional experience. Finally, this study analyzed patients based on their biologically determined sex and does not address differences based on gender identity and related socially determined variables (33-35).

\section{Conclusions}

This is the first study reporting on sex differences in short 
and long-term outcomes following less-invasive LVAD implantation. Our single-center experience suggests that women suffering from end-stage HF can benefit from a less-invasive surgical approach at the time of LVAD implantation. Notwithstanding, this analysis should serve as motivation to further investigate the role of surgical techniques in the context of a more individualized LVAD therapy, taking into account sex differences.

\section{Acknowledgments}

The authors gratefully acknowledge all VAD-coordinators and study nurses for their support in data collection and patients' assistance. We kindly thank Marta Cucchi for the language review.

Funding: None.

\section{Footnote}

Conflicts of Interest: JDS and GD are consultants for Abbott and Medtronic. JSH is consultant for Abbott. LCN received consultant, proctoring and lecture honoraria from Abiomed and lecture honoraria from Abbott, Maquet and Orion, outside the submitted work. Other relationships unrelated to this work exist. RL is consultant for Medtronic and LivaNova, and Advisory Board Member of Eurosets: all honoraria are paid to the University for research support. The other authors have no conflicts of interest to declare.

Open Access Statement: This is an Open Access article distributed in accordance with the Creative Commons Attribution-NonCommercial-NoDerivs 4.0 International License (CC BY-NC-ND 4.0), which permits the noncommercial replication and distribution of the article with the strict proviso that no changes or edits are made and the original work is properly cited (including links to both the formal publication through the relevant DOI and the license). See: https://creativecommons.org/licenses/by-nc-nd/4.0/.

\section{References}

1. Schmitto JD, Pya Y, Zimpfer D, et al. Long-term evaluation of a fully magnetically levitated circulatory support device for advanced heart failure-two-year results from the HeartMate 3 CE Mark Study. Eur J Heart Fail 2019;21:90-7.

2. Chatterjee A, Mariani S, Hanke JS, et al. Minimally invasive left ventricular assist device implantation: optimizing device design for this approach. Expert Rev Med Devices 2020;17:323-30.

3. Zimpfer D, Fiane AE, Larbalestier R, et al. LongTerm Survival of Patients With Advanced Heart Failure Receiving an Left Ventricular Assist Device Intended as a Bridge to Transplantation: The Registry to Evaluate the HeartWare Left Ventricular Assist System. Circ Heart Fail 2020;13:e006252.

4. Teuteberg JJ, Cleveland JC Jr, Cowger J, et al. The Society of Thoracic Surgeons Intermacs 2019 Annual Report: The Changing Landscape of Devices and Indications. Ann Thorac Surg 2020;109:649-60.

5. Kormos RL, Cowger J, Pagani FD, et al. The Society of Thoracic Surgeons Intermacs database annual report: Evolving indications, outcomes, and scientific partnerships. J Heart Lung Transplant 2019;38:114-26.

6. Joshi AA, Lerman JB, Sajja AP, et al. Sex-Based Differences in Left Ventricular Assist Device Utilization: Insights From the Nationwide Inpatient Sample 2004 to 2016. Circ Heart Fail 2019;12:e06082.

7. DeFilippis EM, Truby LK, Garan AR, et al. Sex-Related Differences in Use and Outcomes of Left Ventricular Assist Devices as Bridge to Transplantation. JACC Heart Fail 2019;7:250-7.

8. Ahmed A, Adegbala O, Akintoye E, et al. Gender Differences in Outcomes After Implantation of Left Ventricular Assist Devices. Ann Thorac Surg 2020;109:780-6.

9. Gruen J, Caraballo C, Miller PE, et al. Sex Differences in Patients Receiving Left Ventricular Assist Devices for EndStage Heart Failure. JACC Heart Fail 2020;8:770-9.

10. Potapov E, Schweiger M, Lehmkuhl E, et al. Gender differences during mechanical circulatory support. ASAIO J 2012;58:320-5.

11. Birks EJ, McGee EC Jr, Aaronson KD, et al. An examination of survival by sex and race in the HeartWare Ventricular Assist Device for the Treatment of Advanced Heart Failure (ADVANCE) Bridge to Transplant (BTT) and continued access protocol trials. J Heart Lung Transplant 2015;34:815-24.

12. Magnussen C, Bernhardt AM, Ojeda FM, et al. Gender differences and outcomes in left ventricular assist device support: The European Registry for Patients with Mechanical Circulatory Support. J Heart Lung Transplant 2018;37:61-70.

13. Hsich EM, Naftel DC, Myers SL, et al. Should women receive left ventricular assist device support?: findings from INTERMACS. Circ Heart Fail 2012;5:234-40. 
14. Bogaev RC, Pamboukian SV, Moore SA, et al. Comparison of outcomes in women versus men using a continuous-flow left ventricular assist device as a bridge to transplantation. J Heart Lung Transplant 2011;30:515-22.

15. Blumer V, Mendirichaga R, Hernandez GA, et al. SexSpecific Outcome Disparities in Patients Receiving Continuous-Flow Left Ventricular Assist Devices: A Systematic Review and Meta-analysis. ASAIO J 2018;64:440-9.

16. Morris AA, Pekarek A, Wittersheim K, et al. Gender differences in the risk of stroke during support with continuous-flow left ventricular assist device. J Heart Lung Transplant 2015;34:1570-7.

17. Sherazi S, Kutyifa V, McNitt S, et al. Effect of Gender on the Risk of Neurologic Events and Subsequent Outcomes in Patients With Left Ventricular Assist Devices. Am J Cardiol 2017;119:297-301.

18. Lamba HK, Kherallah RY, Hudson SI, et al. Do Women Have Inferior Outcomes to Men after LVAD Implantation- A Propensity-Matched Comparison. The Journal of Heart and Lung Transplantation 2020. doi: 10.1016/j.healun.2020.01.222.

19. Weymann A, Patil NP, Sabashnikov A, et al. Gender differences in continuous-flow left ventricular assist device therapy as a bridge to transplantation: a risk-adjusted comparison using a propensity score-matching analysis. Artif Organs 2015;39:212-9.

20. Schmitto JD, Molitoris U, Haverich A, et al. Implantation of a centrifugal pump as a left ventricular assist device through a novel, minimized approach: upper hemisternotomy combined with anterolateral thoracotomy. J Thorac Cardiovasc Surg 2012;143:511-3.

21. McGee E Jr, Danter M, Strueber M, et al. Evaluation of a lateral thoracotomy implant approach for a centrifugalflow left ventricular assist device: The LATERAL clinical trial. J Heart Lung Transplant 2019;38:344-51.

22. Wert L, Chatterjee A, Dogan G, et al. Minimally invasive surgery improves outcome of left ventricular assist device surgery in cardiogenic shock. J Thorac Dis 2018;10:S1696-702.

23. Sileshi B, O'Hara BK, Davis ME, et al. Outcomes of Patients Implanted Using a Left Thoracotomy Technique for a Miniaturized Centrifugal Continuous-Flow Pump. ASAIO J 2016;62:539-44.

24. Pasrija C, Sawan MA, Sorensen E, et al. Less invasive left ventricular assist device implantation may reduce right ventricular failure. Interact Cardiovasc Thorac Surg 2019;29:592-8.
25. Sahutoglu C, Turksal E, Bilic U, et al. Anesthetic Management for Left Ventricular Assist Device Implantation Through Left Thoracotomy: Evaluation of On-Pump Versus Off-Pump. Transplant Proc 2017;49:587-92.

26. Mohite PN, Sabashnikov A, Raj B, et al. Minimally Invasive Left Ventricular Assist Device Implantation: A Comparative Study. Artif Organs 2018;42:1125-31.

27. Hanke JS, Rojas SV, Cvitkovic T, et al. First results of HeartWare left ventricular assist device implantation with tunnelling of the outflow graft through the transverse sinus. Interact Cardiovasc Thorac Surg 2017;25:503-8.

28. Maltais S, Anwer LA, Tchantchaleishvili V, et al. Left Lateral Thoracotomy for Centrifugal Continuous-Flow Left Ventricular Assist Device Placement: An Analysis from the Mechanical Circulatory Support Research Network. ASAIO J 2018;64:715-20.

29. Gosev I, Wood K, Ayers B, et al. Implantation of a fully magnetically levitated left ventricular assist device using a sternal-sparing surgical technique. J Heart Lung Transplant 2020;39:37-44.

30. Wagner CE, Bick JS, Kennedy J, et al. Minimally invasive thoracic left ventricular assist device implantation; case series demonstrating an integrated multidisciplinary strategy. J Cardiothorac Vasc Anesth 2015;29:271-4.

31. Mahr C, McGee E Jr, Cheung A, et al. Cost-Effectiveness of Thoracotomy Approach for the Implantation of a Centrifugal Left Ventricular Assist Device. ASAIO J 2020;66:855-61.

32. Mokadam NA, McGee E Jr, Wieselthaler G, et al. Cost of Thoracotomy Approach: An Analysis of the LATERAL Trial. Ann Thorac Surg 2020;110:1512-9.

33. Richie C. Sex, not gender. A plea for accuracy. Exp Mol Med 2019;51:1.

34. Clayton JA, Tannenbaum C. Reporting Sex, Gender, or Both in Clinical Research? JAMA 2016;316:1863-4.

35. Heidari S, Babor TF, De Castro P, et al. Sex and Gender Equity in Research: rationale for the SAGER guidelines and recommended use. Res Integr Peer Rev 2016;1:2.

36. Haberl T, Riebandt J, Mahr S, et al. Viennese approach to minimize the invasiveness of ventricular assist device implantationdagger. Eur J Cardiothorac Surg 2014;46:9916; discussion 996.

37. Schmitto JD, Mariani S, Abicht TO, et al. Expert Consensus Paper: Lateral Thoracotomy for Centrifugal Ventricular Assist Device Implant. Ann Thorac Surg 2020. doi: 10.1016/j.athoracsur.2020.09.063.

38. Feldman D, Pamboukian SV, Teuteberg JJ, et al. The 2013 
International Society for Heart and Lung Transplantation Guidelines for mechanical circulatory support: executive summary. J Heart Lung Transplant 2013;32:157-87.

39. Cook JL, Colvin M, Francis GS, et al. Recommendations for the Use of Mechanical Circulatory Support:

Ambulatory and Community Patient Care: A Scientific Statement From the American Heart Association. Circulation 2017;135:e1145-e1158.

40. Mentzer G, Hsich EM. Heart Failure with Reduced Ejection Fraction in Women: Epidemiology, Outcomes, and Treatment. Heart Fail Clin 2019;15:19-27.

41. Shah P, Ha R, Singh R, et al. Multicenter experience with durable biventricular assist devices. J Heart Lung Transplant 2018;37:1093-101.

42. Meeteren JV, Maltais S, Dunlay SM, et al. A multiinstitutional outcome analysis of patients undergoing left ventricular assist device implantation stratified by sex and race. J Heart Lung Transplant 2017;36:64-70.

43. Kocabeyoglu SS, Kervan U, Sert DE, et al. Is it Possible

Cite this article as: Mariani S, Li T, Bounader K, Boethig D, Schöde A, Hanke JS, Michaelis J, Napp LC, Berliner D, Dogan G, Lorusso R, Haverich A, Schmitto JD. Sex differences in outcomes following less-invasive left ventricular assist device implantation. Ann Cardiothorac Surg 2021;10(2):255-267. doi:10.21037/acs-2020-cfmcs-21 to Implant HeartMate 3 Less Invasively? New Pump, New Approach. Artif Organs 2018;42:1132-8.

44. Salzano A, Demelo-Rodriguez P, Marra A, et al. A Focused Review of Gender Differences in Antithrombotic Therapy. Curr Med Chem 2017;24:2576-88.

45. Campbell NR, Hull RD, Brant R, et al. Different effects of heparin in males and females. Clin Invest Med 1998;21:71-8.

46. Sullivan K, Doumouras BS, Santema BT, et al. Canadian Journal of Cardiology Sex-Based Differences in Heart Failure: Pathophysiology, Risk Factors, Management, and Outcomes. Can J Cardiol 2020. doi: 10.1016/ j.cjca.2020.12.025.

47. Goldstein DJ, Mehra MR, Naka Y, et al. Impact of age, sex, therapeutic intent, race and severity of advanced heart failure on short-term principal outcomes in the MOMENTUM 3 trial. J Heart Lung Transplant 2018;37:7-14. 


\section{INTERMACS 1 patients before Propensity Score Matching}
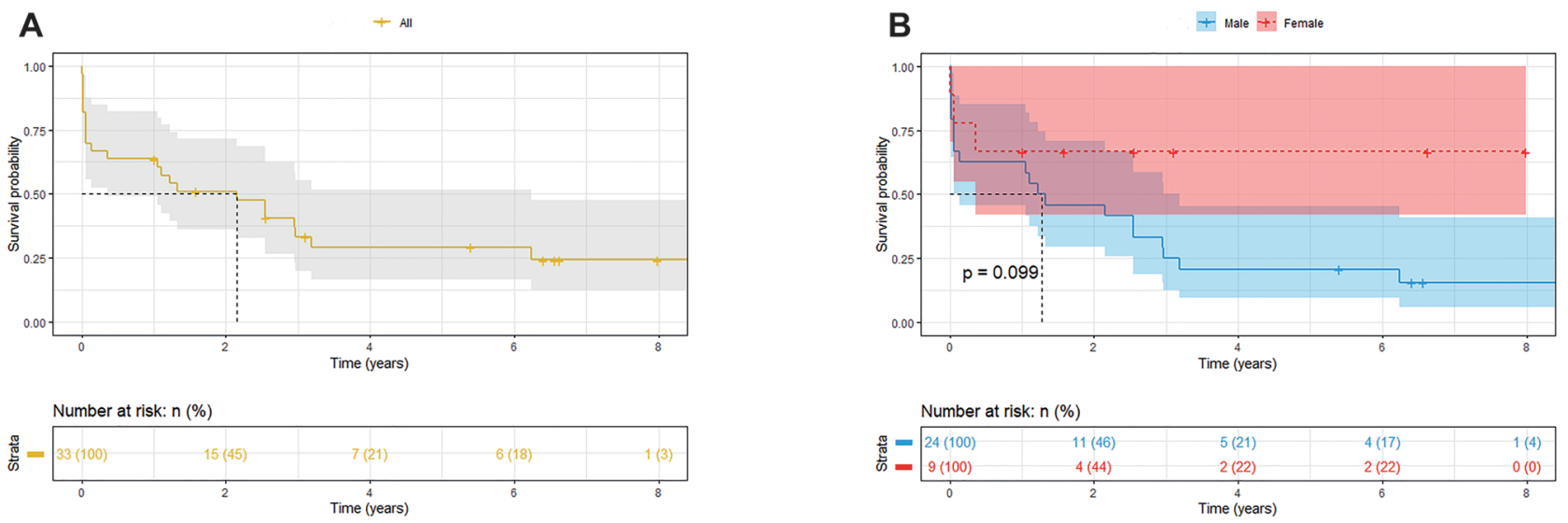

INTERMACS > 1 patients before Propensity Score Matching
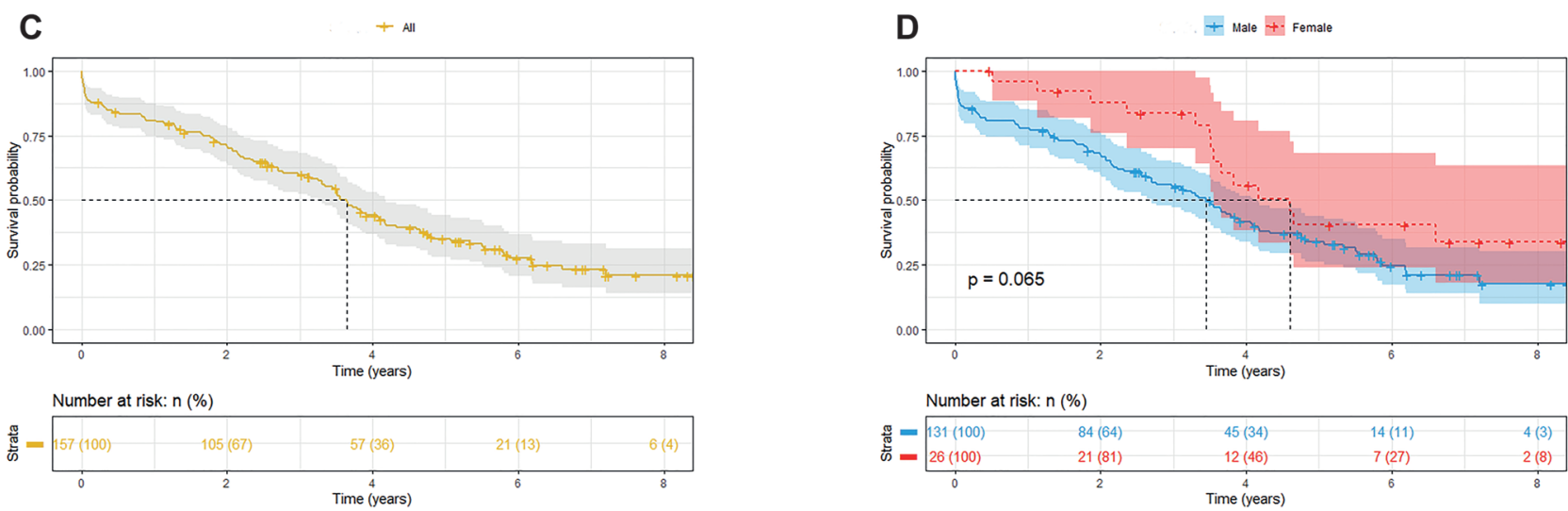

Figure S1 Kaplan-Meier survival curves of the overall cohort $(A, C)$ and by sex $(B, D)$ before propensity score matching and stratified by INTERMACS profile. 